\title{
BMJ Open CLARIFY 2021: explanation and elaboration of the Delphi-based guidelines for the reporting of yoga research
}

\author{
Steffany Moonaz (D) , ${ }^{1}$ Daryl Nault (D) , ${ }^{1}$ Holger Cramer (D) , ${ }^{2}$ Lesley Ward ${ }^{3}$
}

To cite: Moonaz S, Nault D, Cramer $\mathrm{H}$, et al. CLARIFY 2021: explanation and elaboration of the Delphi-based guidelines for the reporting of yoga research. BMJ Open 2021;11:e045812. doi:10.1136/ bmjopen-2020-045812

- Prepublication history for this paper is available online. To view these files, please visit the journal online (http://dx.doi. org/10.1136/bmjopen-2020045812).

Received 14 0ctober 2020 Accepted 07 July 2021
Check for updates

(c) Author(s) (or their employer(s)) 2021. Re-use permitted under CC BY-NC. No commercial re-use. See rights and permissions. Published by BMJ.

${ }^{1}$ Department of Integrative Health Research, Maryland University of Integrative Health, Laurel, Maryland, USA

${ }^{2}$ Department of Internal and Integrative Medicine, Evang, Kliniken Essen-Mitte, Faculty of Medicine, University of Duisburg-Essen, Essen,

Germany

${ }^{3}$ Department of Sport, Exercise \& Rehabilitation, Northumbria University, Newcastle Upon Tyne, UK

Correspondence to Dr Steffany Moonaz; smoonaz@muih.edu

\section{ABSTRACT}

Background Reporting of yoga research often lacks the detail required for clinical application, study replication, summary research and comparative effectiveness studies. Methods To improve the transparency of reporting yoga interventions, and building on the development of previous reporting guidelines, a group of international yoga research stakeholders developed the consensus-based CheckList stAndardising the Reporting of Interventions For Yoga (CLARIFY) guidelines.

Results The 21-item CLARIFY checklist outlines the minimum details considered necessary for high-quality reporting of yoga research. This paper provides a detailed explanation of each of the 21 items of the CLARIFY checklist, together with model examples of how to integrate each item into publications of yoga research. The CLARIFY guideline serves as an extension for existing research reporting guidelines, and is flexible for use across all study designs.

Conclusion We strongly encourage the uptake of these reporting guidelines by researchers and journals, to facilitate improvements in the transparency and utility of yoga research.

\section{INTRODUCTION}

The practice of yoga has increased dramatically in recent years, including its use for management of health conditions. ${ }^{12}$ Published yoga research has kept pace and has shown an exponential rise in volume and more rigorous study designs. ${ }^{34}$ Unfortunately, reporting of yoga interventions is often lacking in transparency, which reduces the potential for evidence-informed practice, study replication, summary research and appropriate policy decisions. ${ }^{5}$ Research reporting guidelines have been instrumental in improving research reporting overall ${ }^{6}$ and guideline extensions have been created for areas such as acupuncture, exercise and herbal products. ${ }^{7-9}$ To date, there are no guidelines for yoga research reporting. In response to that need, our aim was to develop internationally relevant consensus-based reporting guidelines through a Delphi-bases

\section{Strengths and limitations of this study}

- Yoga research reporting is inconsistent which hampers clinical application, summary research and policy decisions.

- No research reporting guidelines have previously been developed to improve yoga research reporting.

- A Delphi panel consisting of 53 international yoga research experts developed consensus for a yoga research reporting guideline over four rounds of online surveys.

- This explanation and elaboration document describes the resulting items with examples from the literature where available.

- This guideline can be used by authors, peerreviewers and journal editors to improve reporting quality for yoga research.

survey of recognised experts in the field of yoga research. This paper presents an in-depth explanation and elaboration of the resultant 21-item CheckList stAndardising the Reporting of Interventions For Yoga (CLARIFY) reporting guidelines, as an exemplar for future research use. CLARIFY is an extension to existing reporting guidelines for randomised controlled trials, observational studies and case reports. This extension will provide guidance for complete and transparent reporting of yoga interventions beyond existing general research reporting guidelines.

\section{METHOD}

A comprehensive description of the Delphi study conducted to develop the CLARIFY guidelines is reported separately in our CLARIFY methodology publication. This study was conducted in accordance with standard Delphi procedures ${ }^{10}{ }^{11}$ and based on previous Delphi research in the field of complementary medicine. ${ }^{12} 13$ 
In brief, 128 authors of international yoga research publications were identified by literature searches conducted by members of the Delphi steering committee and invited to take part in the Delphi study. This strategy was supplemented by snowball recruitment, whereby invitees could recommend other yoga researchers for inclusion as Delphi panellists. In total, 53 panellists were recruited.

The Delphi study was conducted over four rounds. Forty-eight panellists from 14 countries completed round 1 (90.6\%), 43 completed round 2 (90\%), 39 completed round $3(81 \%)$ and 32 completed round $4(67 \%)$. Only those who had completed the previous round could take part in the subsequent round.

Panellists rated individual items on a 5-point Likert scale regarding their importance for inclusion in yoga reporting guidelines. According to a priori criteria, items rated as 'very important' or 'extremely important' by at least $80 \%$ of panellists were automatically included in the guidelines, items rated 'of no importance' or 'of little importance' by at least $50 \%$ of panellists, or rated as 'important', 'very important' or 'extremely important' by less than $75 \%$ of panellists were automatically excluded, and items rated 'important', 'very important' or 'extremely important' by at least $75 \%$ of panellists were forwarded to the subsequent round for rerating. Termination criteria were based on inter-round stability of non-consensus items, whereby items with stable or decreasing ratings were discarded on the premise that consensus was not able to be met.

An initial list of 58 items was generated by the steering committee to be rated for inclusion in yoga reporting guidelines. To align the development of the CLARIFY guidelines with existing international standards, these items were grouped into 10 categories in the survey with reference to relevant established reporting guidelines. ${ }^{7814} \mathrm{An}$ additional 15 items for rating were generated by panellists' free-text comments in round 2 and round 3. Fifteen survey items reached consensus for inclusion in round 1 , two items in round 2, three items in round 3 and one item in round 4. The resulting 21-item CLARIFY guideline is presented in table 1.

\section{Patient and public involvement}

This guideline is not directly related to patient care and no patients were involved in the process at any point. Input in the initial development of the survey was gathered from attendees of the Symposium on Yoga Research annual meeting. The Delphi panellists who served as survey participants were provided with survey findings after each stage of the Delphi process, including the final consensus guideline items. Further detail on panellist involvement can be found in a separate manuscript reporting on survey methodology. Delphi panellists acknowledgement can be found at the end of this document.

\section{RESULTS}

Table 1 presents the 21 CLARIFY items in alignment with their corresponding theme and subtheme. In the elaboration section that follows, each item is explained in detail and quotations provided as exemplars for using the reporting items.

\section{Explanation and elaboration of the 21-item CLARIFY reporting guidelines}

Note: Examples in this section indicated by an asterisk $(*)$ were generated by the authors in the absence of clear and relevant examples in the existing peer-reviewed literature.

CLARIFY item 1: title

In the title, succinctly describe the yoga intervention.

Item 1

Include the word 'yoga' in the publication title.

\section{Explanation}

Yoga is a multifaceted discipline, including a range of practices including physical (asana), breathing (pranayama), concentration (dhyana) and applied yoga philosophy. Depending on the tradition or style of yoga, these practices may be identified by English and/or Sanskrit names. In a research context, yoga interventions may include only one, or a combination of two or more of these practices.

Studies delivering single yoga practices (eg, pranayama only) may not include the term 'yoga' in the title, but instead name the specific yoga practice. Additionally, some studies may use broader terms to describe the physical nature of the yoga practices, such as 'exercise' or 'stretching'. These naming variations make it difficult to find, evaluate, and apply the existing yoga literature.

Author(s) should include the term 'yoga' or 'yogic' in the publication title, to facilitate the identification and retrieval of yoga research by other researchers, clinicians and policymakers. The terms 'yoga' and 'yogic' would be considered appropriate for interventions delivering practices from yogic traditions or grounded in philosophy.

\section{Examples}

1. 'Yoga, physical therapy, or education for chronic low back pain (cLBP): a randomised noninferiority trial. ${ }^{15}$

2. 'Perceptions of hatha yoga among persistently depressed individuals enrolled in a trial of yoga for depression. ${ }^{, 16}$

3. 'Yogic breathing when compared with attention control reduces the levels of pro-inflammatory biomarkers in saliva: a pilot randomised controlled trial. ${ }^{, 17}$

\section{CLARIFY item 2: theory}

Describe any rationale, theory, or goal of the elements essential to the yoga intervention.

\section{Item 2}

Describe why the specific population was included in the study.

\section{Explanation}

Yoga studies should provide a strong rationale for their choice of participant population with regard to the chosen yoga intervention. This applies across all 
Table 1 CLARIFY guideline checklist

\begin{tabular}{|c|c|c|}
\hline No & Theme subtheme & Item \\
\hline 1 & Title & \\
\hline $1 \mathrm{a}$ & Succinctly describe the yoga intervention. & Include the word 'yoga' in the publication title. \\
\hline $2 a$ & $\begin{array}{l}\text { Describe any rationale, theory or goal of the elements } \\
\text { essential to the yoga intervention. }\end{array}$ & Describe why the specific population was included in the study. \\
\hline $3 b$ & & $\begin{array}{l}\text { Describe the duration of yoga practices within the yoga session (eg, } \\
20 \text { min postures, } 10 \text { min breathing). }\end{array}$ \\
\hline 4 & Expertise & \\
\hline $4 a$ & $\begin{array}{l}\text { Describe the expertise, background, and training of those } \\
\text { providing the yoga intervention. }\end{array}$ & Describe the qualifications of the yoga instructor(s). \\
\hline 6 & Dose & \\
\hline $\begin{array}{l}6 a \\
6 b \\
6 c \\
6 d\end{array}$ & $\begin{array}{l}\text { Describe the no of times the yoga intervention was } \\
\text { delivered and over what period, including the no of } \\
\text { sessions, their schedule and their duration and intensity. }\end{array}$ & $\begin{array}{l}\text { Describe the duration of each yoga session (in minutes). } \\
\text { Describe the duration of the yoga intervention (ie, over } 8 \text { weeks). } \\
\text { Describe the frequency of yoga sessions (eg, twice weekly). } \\
\text { Describe the no of yoga sessions. }\end{array}$ \\
\hline 7 & Home practice & \\
\hline $7 a$ & Describe aspects of home practice if any. & Describe the duration and frequency of home practice (if any) \\
\hline 9 & Participant adherence & \\
\hline $\begin{array}{l}9 a \\
9 b\end{array}$ & $\begin{array}{l}\text { If adherence to the yoga intervention was assessed, } \\
\text { describe how and by whom, and what, if any strategies } \\
\text { were used to maintain or improve adherence. }\end{array}$ & $\begin{array}{l}\text { Describe if and how class/session attendance was measured. } \\
\text { Describe any strategies used to promote practice adherence. }\end{array}$ \\
\hline 10 & Instructor fidelity & \\
\hline $10 \mathrm{a}$ & Describe the extent to which the yoga intervention was & Describe the assessment of protocol fidelity \\
\hline $10 b$ & & Describe the reasons for deviation from study plan. \\
\hline $10 \mathrm{c}$ & & $\begin{array}{l}\text { Describe any differences between proposed programme and actual } \\
\text { programme delivery. }\end{array}$ \\
\hline $10 d$ & & Describe when protocol was modified. \\
\hline
\end{tabular}

CLARIFY, CheckList stAndardising the Reporting of Interventions For Yoga.

research designs (eg, feasibility trials, observational research or case reports) and methodologies (qualitative or quantitative). A rationale for the selected population provides important context for the applicability, effectiveness and safety of the yoga intervention to this population, and the interpretation and generalisation of the research findings within the larger body of research evidence.

\section{Examples}

1. 'Exposure to chronic stressors, in concert with other individual and genetic risks for substance use, affect 
neurobiological pathways, increasing both the risk of ineffective stress responses and the risk of substance use to cope with stress (Sinha, 2008). Stress-reduction interventions are crucial for returning citizens to manage this challenging time, in general, and to reduce substance use risk, more specifically (Sinha, 2008) One promising intervention that has been associated with stress reduction is yoga., 18

2. 'Given the limited qualitative literature on yoga for chronic pain conditions, it is unclear if participants with cLBP would have distinct experiences different from those with other types of chronic pain. ${ }^{, 19}$

3. 'We, thus, intended to investigate whether and how specific aspects of spirituality may change within a 6-month intensive yoga practice. To address this question, we chose individuals who were already practising yoga and decided to start a yoga teacher training, which would subject them to a greater intensity, frequency and duration of practice. ${ }^{20}$

\section{CLARIFY item 3: activities}

Describe the yoga practices or activities used in the intervention.

The two items in this section refer to the type and duration of activities included in a yoga intervention. Detailed reporting of these two intervention aspects will improve both the replication and application of research to professional practice. Items may be reported separately or in combination with each other, in various formats flexible to the constraints of journal word limits. These include in-text descriptions or table format, or as online supplemental material. Where applicable, an intervention protocol may be published separately, and referenced in the main study publication.

\section{Item $3 a$}

Describe the type of yoga practices included (eg, postures/ asana, breathing/pranayama, meditation, relaxation).

\section{Explanation}

As stated in Item 1, 'Yoga' is not a standardised concept, but instead covers a range of practices, styles, and modes of delivery. Additionally, the additive or synergistic effects of these various yoga practices is currently unclear. As such, simply describing an intervention as 'yoga', without detailing the practices included, is of limited use for both intervention reproducibility and application of findings. To improve transparency, dissemination, and implementation of yoga research to health professionals, researchers, policy-makers and the general public, yoga practices should be clearly described.

\section{Examples}

1. 'The yoga intervention was for 15 days with two practice sessions each day. Each session was for $120 \mathrm{~min}$. In practice sessions the participants were taught: (1) loosening exercises, (2) physical postures (asanas), (3) breathing techniques (pranayamas) and (4) meditation and relaxation. The details are given in table $1 .^{21}$
2. 'The 75 min yoga classes taught during the 8-week yoga programme will consist of yoga philosophy, warmup exercises, yoga postures (asana), breathing (pranayama) and relaxation practices; with time for group discussion at the beginning and end of each class (table 2). Sixteen yoga postures will be taught over the 8 weeks, with 5-10 postures taught per class (table 3 ). ${ }^{22}$

3. 'The yoga intervention consisted of an initial full-day workshop, followed by 2 weekly 90 min classes of traditional hatha yoga over a 12-week period. The yoga classes were based on integral yoga as developed by Swami Sivananda and an adaptation of the basic yogavidya sequence... eBoxes 1 and 2 show further details of the yoga programme. ${ }^{23}$

Item $3 b$

Describe the duration of yoga practices within the yoga session (eg, $20 \mathrm{~min}$ postures, $10 \mathrm{~min}$ breathing).

\section{Explanation}

To improve understanding and reproducibility of a yoga intervention, the specific amount of time dedicated to each practice should be documented. This includes clarification of changes in duration over the course of an intervention (eg, where if standing postures are increased and seated postures decreased over the course of a progressive intervention). Any individual variability allowed within each practice should also be described.

\section{Examples}

1. 'The class was held over $60 \mathrm{~min}$ in a quiet and dimly lit room, according to the following schedule: 15 min of pranayama (gentle breathing techniques) in the supine position. The pelvis was in a neutral position, and the arms abducted with palms facing toward the ceiling. This was followed by $30 \mathrm{~min}$ of a series of nine modified yoga asanas which comprised of gentle stretching and strengthening exercises specifically targeting the upper body. Each asana was maintained for 5-10 breaths and repeated for five times. The session concluded with 15 min of shavasana. The patient attended three supervised sessions per week for 4 weeks with self-practice on the remaining days. ${ }^{24}$

2. 'The yoga sessions began with an instructor-led breathing practice. The instructor then led participants through yoga poses at a slow but gradually increasing pace, with chairs used as props when needed. Meditation and breathing were followed by chair poses (15-20 min), standing poses (10-15 min), floor poses (15 min) and a supine resting pose (savasana; $10 \mathrm{~min}$ ). A typical class included $20-25$ poses out of 73 possible poses included in the Silver Age Yoga method. ${ }^{25}$

3. 'Each session lasted approximately $75 \mathrm{~min}$, divided into three equal parts: breathing exercises, yogic poses and meditations, education and discussion (details in online supplemental material: YACHT study package_ v1.2.pdf). ${ }^{26}$ 


\section{CLARIFY item 4: expertise}

Describe the expertise, background, and training of those providing the yoga intervention.

Item 4

Describe the qualifications of the yoga instructor(s).

\section{Explanation}

To assist implementation of yoga interventions into clinical or community practice, clarification of the skills necessary to deliver the intervention is required. The credentials, training and/or experience of each yoga instructor delivering the intervention should be detailed. These may include national or international yoga certifications, healthcare licensure, years of teaching experience, prior teaching experience with the study population and/or training for the research study. Any potential impact of instructor variability across multi-site delivery should also be taken into consideration in the study analysis and discussion.

\section{Examples}

1. 'The instructor had more than 30 years of experience as a yoga instructor and had more than 4 years of experience instructing isometric yoga to patients with ME/ CFS (approximately 50 patients in total). ${ }^{27}$

2. 'Two certified prenatal yoga teachers each with at least 4 years of yoga teaching experience were trained by the principal investigator (PI) in the study-specific class. ${ }^{28}$

3. 'All yoga instructors were Registered Yoga Teachers with the Yoga Alliance. A senior yoga instructor, the study primary investigator and other coinvestigators provided training for instructors in the study yoga protocol via didactics, review of the manual and class observation. Instructors met monthly for supervision. ${ }^{29}$

\section{CLARIFY item 5: delivery}

Describe how the yoga intervention was delivered (eg, class, video/audio) and whether it was provided individually or in a group.

\section{Item 5}

Describe the teaching approach and resources used in delivery, including visual demonstration, verbal guidance and/or hands on assistance.

\section{Explanation}

Teaching styles vary markedly both across yoga traditions and between yoga instructors, resulting in variability of delivery within an intervention. Instructors may teach using modelling and/or verbal instruction, remain seated and provide only verbal adjustments, or physically interact with participants by providing hands-on adjustments. Additionally, the pragmatic constraints of cost and time may guide the parameters of intervention delivery. Interventions may be delivered face to face, individually or in groups, or via home practice. Homebased instruction may use delivery platforms including live or recorded audio/video instruction, individual home visits, written material, electronic devices or digital media. Explanations of intervention delivery should detail the mode of delivery (individual, group, home, digital) and amount of instruction delivered via each approach.

\section{Examples}

1. 'Classes were kept small (3-10 participants) to allow for pose modifications as needed for each participant. The same instructor taught all classes with occasional substitution from the PI/yoga instructor (approximately 2-3 times per cohort). 30

2. 'The therapeutic yoga intervention for this study included 1-hour sessions of yoga twice a week for 8 weeks. Yoga was delivered one-on-one by certified yoga therapist. The yoga was delivered using an established protocol but was tailored to meet each individual's needs and abilities. ${ }^{31}$

3. 'In response, we developed and conducted a long-term evaluation of Eyes-Free Yoga, a game that provides solely auditory output using Microsoft Kinect for Windows. We integrated the personalised feedback into a now a fully functional exergame with motivational techniques for which we were able to conduct an 8-week deployment study in the homes of four participants who are blind or low vision. Our system provides four full yoga workouts with descriptive audio instructions. We incorporated relaxing, meditative music in the background to enhance the experience... Additionally, participants used their voice to control Eyes-Free Yoga to avoid the need of a controller while exercising... While holding a pose, the exergame offered verbal adjustments and auditory confirmation. ${ }^{32}$

\section{CLARIFY Item 6: dose}

Describe the number of times the yoga intervention was delivered and over what period, including the number of sessions, their schedule and their duration and intensity.

In addition to transparent reporting of the type of yoga practices included in an intervention, it is important to state the amount or dose, of yoga the participants received. Dosage parameters are a combination of four aspects: the length of each individual yoga session (session duration), the frequency of the yoga session, the duration of the yoga intervention, and the total number of intervention sessions. These four items can be reported separately or combined into a single description of dose.

\section{Item 6a}

Describe the duration of each yoga session (in minutes).

\section{Explanation}

Duration of each yoga session should be stated, including any variability in session duration over the course of the intervention. Describe the duration of all forms of yoga delivery, including face-to-face (individual or group), home practice sessions and audio/video resources. 


\section{Examples}

1. 'The MiYoga intervention was an 8-week programme that consisted of six $90 \mathrm{~min}$ sessions over the initial 6 weeks, followed by two telephone or Skype consultations over the remaining 2 weeks of the programme to help participants integrate MiYoga practices into their everyday life. ${ }^{33}$

2. 'Each week, there was an in-person session that lasted 1.5 hours and included asanas, meditation and other mindfulness-based practices (see online supplemental appendix). ${ }^{, 34}$

3. 'The practices were taught by a certified Yoga instructor volunteers in 9-day camps (2 hours daily) in their respective villages or wards. Subsequently, they were asked to continue the practices daily ( 1 hour) at home, through the use of DVDs. ${ }^{35}$

Item $6 b$

Describe the duration of the yoga intervention (ie, over 8 weeks).

\section{Explanation}

In addition to the duration of each yoga session, the overall duration of the intervention each participant received should be stated. If the intervention is progressive, with different instructional targets at each stage, this should be clearly indicated.

\section{Examples}

1. 'The RISE programme was delivered as a 3-day residential immersion programme at Kripalu. ${ }^{36}$

2. 'Hatha yoga instruction was performed for 26 weeks, 3 times a week, while each session lasted 60-70 min (postures, breathing techniques, meditation) by an experienced yoga instructor. ${ }^{37}$

3. 'Class plans are progressive, with weeks 1-2 focusing on the introduction of basic key yoga practices including a breathing technique, yoga warm-up routine (Pawanmuktasana I), basic supine yoga postures, and body awareness relaxation. Weeks 3-4 introduce the concept of alignment and engagement of core muscles, with the inclusion of seated yoga postures. Weeks 5-6 introduce balance, and basic standing yoga postures; finally, weeks 7-8 introduce strength and stability in standing yoga postures. Postures progress from predominantly supine, to seated, to standing postures over the four class plans (table 3).'22

Item $6 c$

Describe the frequency of yoga sessions (eg, twice weekly).

\section{Explanation}

The frequency of individual sessions should be described. If the frequency is variable or changes over the course of the intervention, this should be noted. (Note: Session frequency refers to the intended frequency of delivery, rather than participant adherence to yoga sessions. Adherence reporting recommendations are outlined in items $7 \mathrm{c}$ and $9 \mathrm{~b}$ ).

\section{Examples}

1. 'The yoga group practised yoga for $75 \mathrm{~min} /$ day, for three consecutive days in a week, over a 12-week period. $^{38}$

2. 'The intervention was administered twice a week for 1.5 hours of duration per session for 8 weeks, and one make up class was available. ${ }^{, 39}$

3. 'Both groups had supervised practices at the centre for 40 min daily (6 days/week) after physiotherapy (20 min) for 2 weeks. ${ }^{40}$

\section{Item 6d}

Describe the number of yoga sessions.

\section{Explanation}

In some studies, the total number of yoga sessions delivered in an intervention is obvious, based on standardised session frequency and intervention duration. For example, twice-weekly sessions delivered over 8 weeks clearly delivers 16 sessions. without the need to state that explicitly. However, in interventions where session frequency is variable, the total number or range, of sessions should be provided. For example, if an intervention delivers once-weekly sessions for 8 weeks, followed by 1-2 sessions per month for 3 months, authors should state that the intervention ranges from 11 to 14 sessions. In pragmatic study designs which do not prespecify the number of sessions provided, such as retrospective observational or case report manuscripts, this item may be fulfilled by reporting the number of sessions attended by the participant(s).

\section{Examples}

1. 'Participants then completed a single private and individually taught standardised 80 min yoga session.' ${ }^{41}$

2. 'During the 10-week intervention period, participants in the yoga group attended a weekly, teacher-led, 90 min yoga class. Participants in the control group were assigned to a yoga wait list for 10 weeks and asked to perform some other type of physical activity for $90 \mathrm{~min}$ each week.' ${ }^{42}$

3. 'Participants assigned to the intervention group underwent a 1-hour laughter therapy session every 2 weeks over the course of 7 weeks, with each participant receiving a total of four sessions. ${ }^{43}$

\section{CLARIFY item 7: home practice}

Describe aspects of home practice if any.

When home practice is encouraged or included in a yoga study, it becomes part of the overall intervention. As such, it needs to be described in sufficient detail to allow intervention replication and application of trial results. Where applicable, this should include home practice dose and adherence measures during the intervention period, and recommendations for yoga practice occurring during any follow-up period. 
Item $7 a$

Explanation

To determine the total dosage of yoga delivered during an intervention, it is important to include any home practice parameters. If home practice is recommended during the intervention period, instructions for the frequency (how often) and duration (how long) of home practice sessions should be included in the manuscript, as well as any specific instructions or resources provided.

\section{Examples}

1. 'Yoga participants received a home practice manual recommending 15-20 min of yoga home practice on days that instructor-led sessions were not held."44

2. 'Thirty minutes of daily home practice, facilitated by a DVD, a manual and take-home yoga supplies, was strongly encouraged. ${ }^{15}$

3. 'Home practice consisted of a 20-min guided relaxation, based on the relaxation technique practised in the group sessions. A CD, recorded by the yoga instructor, was provided. Participants were asked to practise three times per week, at a time and day of their choice. $^{45}$

Item $7 b$

Describe if and how adherence to home practice was measured.

\section{Explanation}

To determine the acceptability and dosage of home practice in yoga research it is important to record participants' adherence to any home practice recommendations. If adherence to home practice was measured, the methods for measurement should be described. These may include, for example, practice diaries, video recording of home practice sessions, use of a practice tracking app, or oral self-report. If home practice was recommended but not recorded, this should be stated.

\section{Examples}

1. 'The patients were also asked to keep a 'yoga diary' in which they recorded the amount of time they practised and any questions they had during the practice. On the day of the visit, the yoga instructor and the patient's doctors checked the diary. ${ }^{46}$

2. 'Participants were instructed on the use of the camera and asked to videotape their usual home yoga practice with a video camera, note the number of minutes practised each time on a log sheet, and include comments associated with their home practice for 1 week. The average frequency and duration of yoga practice and qualitative data on the characteristics of yoga practice including poses and sequences practised, types of tools used, and settings in which yoga practice took place, were analysed. ${ }^{47}$

3. 'Adherence to home practice in the previous week was verbally reported to the yoga instructor at the beginning of each session, and barriers and adherers to home practice were discussed among the group. ${ }^{22}$
Item 7c

Report whether yoga was available to participants during the follow-up period (if relevant), and list any recommendations made for home practice dose.

\section{Explanation}

Some yoga studies include a follow-up period after the yoga intervention has ended, whereby participants may be encouraged to continue yoga practice at home. If so, any recommendations made for continued practice during this period should be described in the manuscript, including frequency and duration of practice and specific practice instructions if relevant.

\section{Examples}

1. 'After the completion of training, participants in the treatment group will be requested to continue the practice at home once every morning for next 90 days. ${ }^{48}$

2. 'Those participants who had completed the 8-week YoA intervention (see description on the following page) and the second assessment were contacted for follow-up at 3 months, without any supervision or additional contact between the second and the third assessments. During this period, they continued with their usual medical care and had been encouraged to continue with their daily yoga practice using the same professionally produced DVD and CDs as they used in the treatment phase of the study protocol. ${ }^{49}$

3. 'Patients were asked to continue daily yoga practice at home. For this purpose, patients received a manual describing and depicting three basic standing postures (mountain pose, standing half-forward bend (at wall), and warrior pose II) and three basic sitting postures (Bharadvaja's twist, prosperous pose without and with spinal twist). ${ }^{50}$

\section{CLARIFY item 8: protocol modifications}

If the delivery of the yoga intervention differed from that described in the protocol, please describe the changes.

Interventions are not always delivered as planned, for many reasons. Any changes to the original content and/ or delivery of the intervention should be clearly described in the research outputs. This information should include the timing and justification of the protocol changes, so the information may be considered in interpretation of the study findings.

\section{Item $8 a$}

Describe any changes to the yoga protocol during the study.

\section{Explanation}

Changes to trial protocols are currently included in established reporting guidelines. ${ }^{51}$ This current item refers specifically to changes made in the predetermined yoga intervention protocol during the study. Changes may include modification or removal of specific yoga practices from the intervention, adjustments to intervention 
delivery, or amendments to the yoga dose (frequency, intensity, duration).

\section{Examples}

1. Following week 4, Chakrasana (Wheel Pose) was removed from the intervention due to several reports of increased pain by participants following that posture.*

2. Based on participant feedback, we provided a simpler breathing practice (Dirga Swasam) beginning with the second cohort as an alternative to Nadi Shodhana (Alternate Nostril Breathing).*

3. No changes were made to the yoga protocol during the intervention.*

\section{Item $8 b$}

Describe the rationale for changes to the yoga protocol during the study.

\section{Explanation}

Any changes to the yoga protocol during the study must be sufficiently justified with a clear rationale. Such rationale may be clinical (eg, reports of joint pain), logistical (eg, site transportation schedule changed) or research related (eg, IRB required removal of chanting practice).

\section{Examples}

1. 'Prior to obtaining Institutional Review Board clearance, instructions were given to remove 'spiritual' aspects of yoga from the clinical trial. It was not immediately clear which would be considered cultural versus 'spiritual' practices. The decision was ultimately made that no music would be played, there would be no chanting 'OM' or bowing to say 'Namaste,' and the Sanskrit names for yoga poses would not be used. $^{52}$

2. 'One participant was not willing to attend any classes because of discomfort with groups but agreed to practice at home. Another individual avoided the Svasana component of the protocol due to a perceived conflict with his religion (Jehovah's Witness). ${ }^{53}$

3 . Because of a change in the city bus route and participant reliance on public transportation, classes were moved from the local community centre to the hospital fitness centre in late April of 2018.*

\section{Item $8 \mathrm{C}$}

Describe when the protocol was modified.

\section{Explanation}

It is important to state when modifications to the intervention protocol are made. Changes made prior to the start of a yoga intervention will affect all participants alike, while changes made during the intervention may mean some participants receive a different version of an intervention. This in turn could impact efficacy and safety outcomes depending on which version of the intervention participants received.

\section{Examples}

1. While not in the original yoga protocol, chair poses were introduced during the first class.*

2. Class duration was shortened from $75 \mathrm{~min}$ to $60 \mathrm{~min}$ beginning in week 2 of the second cohort.*

3. The order of practices changed in October 2014, with the addition of a new interventionist.*

\section{CLARIFY item 9: adherence}

If adherence to the yoga intervention was assessed, describe how and by whom, and what, if any strategies were used to maintain or improve adherence.

Intervention adherence is an indicator of participant satisfaction and acceptability, and various strategies may be used to promote adherence in physical and behavioural interventions. Monitoring adherence, in turn, enables the calculation of the actual, rather than the prescribed, intervention dose a participant received, to establish thresholds of intervention effectiveness.

\section{Item 9a}

Describe any strategies used to promote practice adherence.

\section{Explanation}

Strategies are often included in exercise-based interventions to promote adherence to both class-based and home-based practice. These strategies may be used during the active phase of the intervention and/or during a follow-up period. Any methods to encourage adherence to yoga practice should be described. This may include incentives for class attendance, delivery of classes at premises accessible by public transport, discussion of barriers and facilitators to home practice during yoga classes, and the provision of materials for home practice (eg, props, manual, videos).

\section{Examples}

1. 'Class attendance and home yoga practice were encouraged among those not meeting the study practice goals by email and/or telephone counselling. Motivational interviewing techniques were used to encourage class attendance and home yoga practice. These included discussing barriers to class attendance and home practice, identifying potential solutions to the barriers, and setting class attendance and home practice goals. $^{, 54}$

2. 'Patients were randomised to a yoga intervention or control group; the patients in the yoga arm were taught the 10 min seated yoga practice, were given an explanatory DVD and a fold-out pocket guide to encourage adherence at home, and were instructed to incorporate the practice as often as they could. ${ }^{, 55}$

3. 'The patients were advised to continue the practices at home for next 3 months. Telephonic reminders to patients or caregivers were used to encourage adherence. ${ }^{56}$ 
Item $9 b$

Describe if and how class/session attendance was measured.

\section{Explanation}

Ways in which participant attendance at yoga sessions was measured or recorded should be described. When attending classes in person, attendance might be documented by the instructor, or via a sign-in sheet. In the case where yoga is not delivered face to face (eg, via video), other strategies for measuring attendance might be used.

\section{Examples}

1. 'Attendance to facility-based classes was directly monitored and tracked through attendance sheets. ${ }^{54}$

2. 'Class attendance, the name of yoga instructor, home practice and reported side effects are recorded during each class session. ${ }^{57}$

3. 'The studio uses the MindBody business software to develop practitioner accounts and track studio attendance. With consent, practitioners' attendance data were pulled from their accounts for the previous 12 months. ${ }^{58}$

\section{CLARIFY item 10: fidelity}

Describe the extent to which the yoga intervention was delivered as planned.

Fidelity refers to the extent to which a pre-defined plan or intervention is reproduced. Fidelity to the wider study protocol is already covered in established reporting guidelines. ${ }^{59}$ The following three items are concerned specifically with fidelity to the pre-determined yoga intervention protocol, including the description and rationale for why these deviations occurred.

\section{Item $10 \mathrm{a}$}

Describe the assessment of protocol fidelity.

\section{Explanation}

The assessment of yoga instructor fidelity to delivering a pre-defined yoga intervention protocol should be detailed in the research publication. Fidelity monitoring may include independent observation, self-report of the yoga instructor or audio/visual recording of a yoga session for later review. Measurement of fidelity may be quantitative (as with a checklist) and/or qualitative (as with written notes by the assessor).

\section{Examples}

1. 'Using audio recordings and a structured tool, yoga supervisors Dr Tremont and Mr Gillette rated a subset of 55 classes throughout the duration of the study for instructor manual fidelity. Fidelity was excellent for class content (mean fidelity $=95 \%$ ) and teaching style (mean fidelity $=94 \%) .{ }^{60}$

2. 'An expert prenatal yoga instructor who helped develop the manual rated $>20 \%$ of PYP classes on manual fidelity using an 18-item checklist. Fidelity was over $80 \%$ for all classes, with average $=92 \%{ }^{, 61}$
3. 'Fidelity: The consultants reviewed $10 \%$ of the videotaped sessions.' 62

Item 10b

Describe any differences between the proposed programme and actual programme delivery.

\section{Explanation}

To ensure accurate analysis of intervention effectiveness and safety, any differences between the predefined yoga intervention protocol and the intervention actually delivered by the yoga instructor should be reported. If deviations from the proposed programme occurred in only a subset of participants (eg, only those in a specific class or those practising with a specific yoga teacher) this should also be detailed. If there were no protocol deviations, this should be stated.

\section{Examples}

1. 'There were no deviations from the manualised yoga protocol. Any changes were allowed within predetermined guidelines to account for individual differences in comorbidities or injuries. ${ }^{, 63}$

2. Ambient music was played during yoga sessions, which the research team was unaware of until exit interviews were conducted.*

3. The yoga instructor at one of the study sites had replaced alternate nostril breathing with a deep breathing technique.*

Item 10c

Describe the reasons for deviation from study plan.

\section{Explanation}

If the actual intervention delivered by the yoga instructor deviates from the predefined intervention protocol, reasons for this deviation should be listed. Reasons may include those related to the yoga teacher (eg, intentional or unintentional non-adherence to the protocol), participants (eg, unwillingness or inability of participants to follow the programme) or logistics (eg, unavailability of treatment faculties or transport).

\section{Examples}

1. While the yoga classes were intended for a duration of $60 \mathrm{~min}$, another class was scheduled to use the space immediately after which disrupted the closing class activities. The instructor opted to shorten class by 5 min in order to avoid this disruption and protect the relaxation experience of study participants.*

2. Floor-based poses were not included by one yoga instructor, due to nervousness on the part of participants in that class about getting up from the floor. Seated poses were offered in a chair instead.*

3. According to the yoga protocol, Tree Pose should have been instructed using a chair for support and safety. Since chairs were not available at the intervention site, instruction was provided for participants to use the wall instead.* 


\section{DISCUSSION}

Four rounds of a Delphi survey completed by an international panel of yoga experts has generated a 21-item consensus guideline for the complete and transparent reporting of yoga research. Of the initial 58 items presented to panellists, 13 items were rejected by panellists due to consensus of being of low importance, 27 items did not achieve consensus, and 3 items were added due to panellist input and subsequent consensus. As such, this 21-item guideline is presented as the 'minimum' intervention detail that should be reported in any yoga research, and researchers are encouraged to include additional information as applicable for optimal transparency of their research.

The CLARIFY guidelines address concerns among researchers that journal word limits may limit how extensively research can be reported. The 21 CLARIFY items are comparable in number to other reporting extensions in integrative health research. ${ }^{764}$ Additionally, many of the items may be reported in the form of tables and/ or online supplemental material, without impacting the article word limits.

Peer-reviewed journals that publish yoga research should recommend use of the CLARIFY guideline for both authors and peer-reviewers in ensuring greater research transparency. Future research should assess uptake of the CLARIFY, and adherence to these recommendations in published manuscripts both before and after their release.

\section{Strengths and limitations}

This study was strengthened by participation by an international panel of research experts including those with diverse roles within research teams. There was a high level of retention across four rounds of Delphi surveys, in line with existing Delphi research. ${ }^{65}$ This study adhered strictly to Delphi procedures resulting in a guideline that fills a gap in the literature and can be applied across study designs. One limitation of this study was that, while the panellists represented 14 countries across 5 continents, the study team was Eurocentric in make-up. Additionally, it is worth noting that several items in the list did not have any easily identifiable examples and yet were deemed important for intervention transparency by the expert panellists. This highlights a gap in current reporting practice which the CLARIFY guidelines will effectively address, raising the quality of reporting and subsequent dissemination of research findings.

\section{CONCLUSION}

It is our recommendation that the CLARIFY guidelines be used by researchers in drafting research manuscripts, peer-reviewers in evaluating research transparency and journal editors as guidance to both above parties. Increased reporting quality in yoga research will ultimately benefit future research, clinical recommendations, policy decisions and patient outcomes.
Acknowledgements The authors acknowledge the following CLARIFY panelists and pilot testers for their service in contribution to this guideline through all stages of the Delphi process: Timothy Avery, Kylie Barr, Susan Bartlett, Peter Bayley, Christiane Brems, Yvonne Colgrove, Michael Demanincor, Jeffery Dusek, Steriani Elavsky, Erik Groessl, Marshall Hagins, Zoe Hewett, Kim Innes, Sara Lazar, Casey Mace-Firebaugh, Romy Lauche, Megan Maiya, Roseny Flávia Martins, Kimberly Middleton, Jani Mikkonen, Charlene Muhammad, James Newham, Barry Oken, JuYoung Park, Abbas Rakhshani, Arlene Schmid, Karen Sherman, Vinoy Singh, Chris Streeter, Marlysa Sullivan, Anne Tiedemann, Laura Todd, Eda Tonga, Marieke Van Puymbroeck and those who wished to remain anonymous.

Contributors SM: study conception, data analysis and interpretation, manuscript drafting and revision, final approval, agreement to authorship. DN: data acquisition, analysis and interpretation, manuscript drafting and revision, final approval, agreement to authorship. HC: data analysis and interpretation, manuscript revision, final approval, agreement to authorship. LW: study design, data analysis and interpretation, manuscript drafting and revision, final approval, agreement to authorship.

Funding Research reported in this publication was partially supported by the National Center for Complementary and Integrative Health of the National Institutes of Health under award number R24 AT001293.

Disclaimer The content is solely the responsibility of the authors and does not necessarily represent the official views of the National Institutes of Health. The funders had no role in study design, data collection and analysis, decision to publish, or preparation of the manuscript.

Competing interests None declared.

Patient and public involvement Patients and/or the public were not involved in the design, or conduct, or reporting, or dissemination plans of this research.

Patient consent for publication Obtained.

Ethics approval The study was approved by the Institutional Review Board at the Maryland University of Integrative Health (07.M00.06.18.2). All participants provided informed consent.

Provenance and peer review Not commissioned; externally peer reviewed.

Data availability statement No data are available. Data will be reported in a separate methodology paper.

Open access This is an open access article distributed in accordance with the Creative Commons Attribution Non Commercial (CC BY-NC 4.0) license, which permits others to distribute, remix, adapt, build upon this work non-commercially, and license their derivative works on different terms, provided the original work is properly cited, appropriate credit is given, any changes made indicated, and the use is non-commercial. See: http://creativecommons.org/licenses/by-nc/4.0/.

\section{ORCID iDs}

Steffany Moonaz http://orcid.org/0000-0003-3143-1631

Daryl Nault http://orcid.org/0000-0002-7441-9256

Holger Cramer http://orcid.org/0000-0002-3640-8046

\section{REFERENCES}

1 Ding D, Stamatakis E. Yoga practice in England 1997-2008: prevalence, temporal trends, and correlates of participation. BMC Res Notes 2014;7:172.

2 Cramer H, Ward L, Steel A, et al. Prevalence, patterns, and predictors of yoga use: results of a U.S. nationally representative survey. Am J Prev Med 2016;50:230-5.

3 Jeter PE, Slutsky J, Singh N, et al. Yoga as a therapeutic intervention: a bibliometric analysis of published research studies from 1967 to 2013. J Altern Complement Med 2015;21:586-92.

4 Cramer H, Langhorst J, Dobos G, et al. Associated factors and consequences of risk of bias in randomized controlled trials of yoga: a systematic review. PLoS One 2015;10:e0144125.

5 Ward L, Stebbings S, Cherkin D, et al. Components and reporting of yoga interventions for musculoskeletal conditions: a systematic review of randomised controlled trials. Complement Ther Med 2014;22:909-19.

6 Mercieca-Bebber R, Rouette J, Calvert M, et al. Preliminary evidence on the uptake, use and benefits of the CONSORT-PRO extension. Qual Life Res 2017;26:1427-37.

7 MacPherson $\mathrm{H}$, Altman DG, Hammerschlag R, et al. Revised standards for reporting interventions in clinical trials of acupuncture 
(stricta): extending the CONSORT statement. PLoS Med 2010; 7: 1000261

8 Slade SC, Dionne CE, Underwood M, et al. Consensus on exercise reporting template (CERT): modified Delphi study. Phys Ther 2016:96:1514-24

9 Gagnier JJ, Boon $\mathrm{H}$, Rochon $\mathrm{P}$, et al. Reporting randomized, controlled trials of herbal interventions: an elaborated consort statement. Ann Intern Med 2006;144:364-7.

10 Hasson F, Keeney S, McKenna H. Research guidelines for the Delphi survey technique. J Adv Nurs 2000;32:1008-15.

11 Keeney S, Hasson F, McKenna H. Consulting the oracle: ten lessons from using the Delphi technique in nursing research. J Adv Nurs 2006;53:205-12.

12 Ward L, Stebbings S, Sherman KJ, et al. Establishing key components of yoga interventions for musculoskeletal conditions: a Delphi survey. BMC Complement Altern Med 2014;14:196.

13 Goldenberg JZ, Ward L, Day A, et al. Naturopathic approaches to irritable bowel syndrome-a Delphi study. J Altern Complement Med 2019;25:227-33.

14 Hoffmann TC, Glasziou PP, Boutron I, et al. Better reporting of interventions: template for intervention description and replication (TIDieR) checklist and guide. BMJ 2014;348:91687.

15 Saper RB, Lemaster C, Delitto A, et al. Yoga, physical therapy, or education for chronic low back pain: a randomized noninferiority trial. Ann Intern Med 2017;167:85-94.

16 Uebelacker LA, Kraines M, Broughton MK, et al. Perceptions of hatha yoga amongst persistently depressed individuals enrolled in a trial of yoga for depression. Complement Ther Med 2017;34:149-55.

17 Twal WO, Wahlquist AE, Balasubramanian S. Yogic breathing when compared to attention control reduces the levels of pro-inflammatory biomarkers in saliva: a pilot randomized controlled trial. BMC Complement Altern Med 2016;16:294.

18 Wimberly AS, Engstrom M, Layde M, et al. A randomized trial of yoga for stress and substance use among people living with HIV in reentry. J Subst Abuse Treat 2018:94:97-104.

19 Keosaian JE, Lemaster CM, Dresner D, et al. 'We're all in this together': a qualitative study of predominantly low income minority participants in a yoga trial for chronic low back pain. Complement Ther Med 2016;24:34-9.

20 Büssing A, Hedtstück A, Khalsa SBS, et al. Development of specific aspects of spirituality during a 6-month intensive yoga practice. Evid Based Complement Alternat Med 2012;2012:1-7.

21 Telles S, Sharma SK, Gupta RK, et al. The impact of yoga on teachers' self-rated emotions. BMC Res Notes 2019;12:680.

22 Ward L, Stebbings S, Athens J, et al. Yoga for pain and sleep quality in rheumatoid arthritis: study protocol for a pilot randomized controlled trial. Physical Therapy Reviews 2014;19:266-76.

23 Cramer H, Thoms MS, Anheyer D. Yoga bei Frauen MIT zentraler Adipositas: Eine randomisierte kontrollierte studie. Dtsch Arztebl Int 2016;113:645-52.

24 Dangi AA, Aurangabadkar SK, Deo MV. Effect of a structured yoga program on fatigue, depression, cardiorespiratory fitness, and quality of life in a postmenopausal breast cancer Survivor. Int $J$ Yoga 2018;11:255.

25 Groessl EJ, Maiya M, Schmalzl L, et al. Yoga to prevent mobility limitations in older adults: feasibility of a randomized controlled trial. BMC Geriatr 2018;18:306.

26 Tillin T, Tuson C, Sowa B, et al. Yoga and cardiovascular health trial (yacht): a UK-based randomised mechanistic study of a yoga intervention plus usual care versus usual care alone following an acute coronary event. BMJ Open 2019;9:e030119.

27 Takakura S, Oka T, Sudo N. Changes in circulating microRNA after recumbent isometric yoga practice by patients with myalgic Encephalomyelitis/Chronic fatigue syndrome: an explorative pilot study. Biopsychosoc Med 2019;13:29.

28 Holden SC, Manor B, Zhou J. Prenatal yoga for back pain, balance, and maternal wellness: a randomized, controlled pilot study. Glob Adv Health Med 2019;8:216495611987098.

29 Uebelacker LA, Weinstock LM, Battle CL, et al. Treatment credibility, expectancy, and preference: prediction of treatment engagement and outcome in a randomized clinical trial of hatha yoga vs. health education as adjunct treatments for depression. $J$ Affect Disord 2018;238:111-7.

30 Middleton KR, Ward MM, Haaz Moonaz S, et al. Feasibility and assessment of outcome measures for yoga as self-care for minorities with arthritis: a pilot study. Pilot Feasibility Stud 2018;4:1-11.

31 Schmid AA, Miller KK, Van Puymbroeck M, et al. Feasibility and results of a case study of yoga to improve physical functioning in people with chronic traumatic brain injury. Disabil Rehabil 2016;38:914-20.
32 Rector K, Vilardaga R, Lansky L, et al. Design and real-world evaluation of Eyes-Free yoga: an Exergame for blind and Low-Vision exercise. ACM Trans Access Comput 2017;9.

33 Mak C, Whittingham K, Cunnington R, et al. Effect of mindfulness yoga programme MiYoga on attention, behaviour, and physical outcomes in cerebral palsy: a randomized controlled trial. Dev Med Child Neurol 2018;60:922-32.

34 Lazaridou A, Koulouris A, Devine JK, et al. Impact of daily yogabased exercise on pain, catastrophizing, and sleep amongst individuals with fibromyalgia. J Pain Res 2019;12:2915-23.

35 Nagarathna R, Tyagi R, Kaur G, et al. Efficacy of a validated yoga protocol on dyslipidemia in diabetes patients: NMB-2017 India trial. Medicines 2019;6:100.

36 Trent NL, Borden S, Miraglia M. Improvements in psychological and occupational well-being following a brief Yoga-Based program for education professionals. Glob Adv Health Med 2019;8:216495611985685.

37 Azami M, Hafezi Ahmadi MR, YektaKooshali MH, et al. Effect of yoga on lipid profile and C-reactive protein in women. Int J Prev Med 2019;10:81.

38 Telles S, Sharma SK, Kala N, et al. Twelve weeks of yoga or nutritional advice for centrally obese adult females. Front Endocrinol 2018;9:466

39 Evans S, Seidman L, Sternlieb B, et al. Clinical case report: yoga for fatigue in five young adult survivors of childhood cancer. J Adolesc Young Adult Oncol 2017;6:96-101.

40 Ebnezar J, Nagarathna R, Bali Y, et al. Effect of an integrated approach of yoga therapy on quality of life in osteoarthritis of the knee joint: a randomized control study. Int J Yoga 2011;4:55.

41 Mackenzie MJ, Carlson LE, Paskevich DM, et al. Associations between attention, affect and cardiac activity in a single yoga session for female cancer survivors: an enactive neurophenomenology-based approach. Conscious Cogn 2014;27:129-46.

42 Kerekes N, Fielding C, Apelqvist S. Yoga in correctional settings: a randomized controlled study. Front Psychiatry 2017;8:204.

43 Morishima T, Miyashiro I, Inoue N, et al. Effects of laughter therapy on quality of life in patients with cancer: an open-label, randomized controlled trial. PLoS One 2019;14:e0219065.

44 Groessl EJ, Liu L, Chang DG, et al. Yoga for military veterans with chronic low back pain: a randomized clinical trial. Am J Prev Med 2017:53:599-608.

45 Ward L, Stebbings S, Athens J, et al. Yoga for the management of pain and sleep in rheumatoid arthritis: a pilot randomized controlled trial. Musculoskeletal Care 2018;16:39-47.

46 Oka T, Tanahashi T, Sudo N, et al. Changes in fatigue, autonomic functions, and blood biomarkers due to sitting isometric yoga in patients with chronic fatigue syndrome. Biopsychosoc Med 2018;12:3

47 Cheung C, Justice C, Peden-McAlpine C. Yoga adherence in older women six months post-osteoarthritis intervention. Glob Adv Health Med 2015;4:16-23.

48 Dhungana RR, Khanal MK, Joshi S, et al. Impact of a structured yoga program on blood pressure reduction among hypertensive patients: study protocol for a pragmatic randomized multicenter trial in primary health care settings in Nepal. BMC Complement Altern Med 2018;18:207

49 Carson JW, Carson KM, Jones KD, et al. Follow-Up of yoga of awareness for fibromyalgia: results at 3 months and replication in the wait-list group. Clin J Pain 2012;28:804-13.

50 Cramer $\mathrm{H}$, Lauche $\mathrm{R}$, Hohmann $\mathrm{C}$, et al. Yoga for chronic neck pain: a 12-month follow-up. Pain Med 2013;14:541-8.

51 Moher D, Hopewell S, Schulz KF, et al. Consort 2010 explanation and elaboration: updated guidelines for reporting parallel group randomised trials. Int J Surg 2012;10:28-55.

52 Middleton KR, Andrade R, Moonaz SH, et al. Yoga research and spirituality: a case study discussion. Int $J$ Yoga Therap 2015;25:33-5.

53 Saper RB, Sherman KJ, Cullum-Dugan D, et al. Yoga for chronic low back pain in a predominantly minority population: a pilot randomized controlled trial. Altern Ther Health Med 2009;15:18-27.

54 Littman AJ, Bertram LC, Ceballos R, et al. Randomized controlled pilot trial of yoga in overweight and obese breast cancer survivors: effects on quality of life and anthropometric measures. Support Care Cancer 2012;20:267-77.

$55 \mathrm{~S}$ Mullur R, Ames D. Impact of a 10 minute seated yoga practice in the management of diabetes. J Yoga Phys Ther 2016;06.

56 Bangalore NG, Varambally S, Ng B. Yoga therapy for schizophrenia Int J Yoga 2012;5:85.

57 Middleton KR, Ward MM, Haaz S, et al. A pilot study of yoga as selfcare for arthritis in minority communities. Health Qual Life Outcomes 2013;11:55. 
58 Harden SM, Steketee AM, Kelliher R, et al. Using a studio-academic partnership to advance public health within a pragmatic yoga setting. J Prim Care Community Health 2019;10:215013271987462.

59 Boutron I, Altman DG, Moher D, et al. Consort statement for randomized trials of nonpharmacologic treatments: a 2017 update and a consort extension for nonpharmacologic trial Abstracts. Ann Intern Med 2017;167:40-7.

60 Uebelacker LA, Tremont G, Gillette LT, et al. Adjunctive yoga $v$. health education for persistent major depression: a randomized controlled trial. Psychol Med 2017;47:2130-42.

61 Uebelacker LA, Battle CL, Sutton KA, et al. A pilot randomized controlled trial comparing prenatal yoga to perinatal health education for antenatal depression. Arch Womens Ment Health 2016;19:543-7.
62 Adair M, Murphy B, Yarlagadda S, et al. Feasibility and preliminary efficacy of tailored yoga in survivors of head and neck cancer: a pilot study. Integr Cancer Ther 2018;17:774-84.

63 Evans S, Seidman LC, Lung K, et al. Yoga for teens with irritable bowel syndrome: results from a mixed-methods pilot study. Holist Nurs Pract 2018;32:253-60.

64 Cheng C-W, Wu T-X, Shang H-C, et al. Consort extension for Chinese herbal medicine formulas 2017: recommendations, explanation, and elaboration. Ann Intern Med 2017;167:112-21.

65 Gargon E, Crew R, Burnside G, et al. Higher number of items associated with significantly lower response rates in COS Delphi surveys. J Clin Epidemiol 2019;108:110-20. 